\title{
Autologous Gene Therapy - A Proactive Approach to Cancer Critique Irfan Ahmad ${ }^{1 *}$ and Reshman Shahin ${ }^{2}$
}

${ }^{1}$ Amity Institute of Biotechnology, Amity University, India

${ }^{2}$ Department of Biochemistry, Allahabad University, India

\begin{abstract}
Cancer is one of the most prevalent diseases that affect the major population with each year the frequency is increasing exponentially. Patients with diagnosed cancer are generally treated with the conventional therapies like radiation therapy, chemotherapy. However the survival rate and the life span are known to prolonged, these therapies are quite problematic let alone their detrimental side effects. Gene therapy on the other hand is quite promising since the body's immune response is not compromised while targeting the cancerous cell. While getting further insights into the complex pathways and mechanisms associated with the development of malignant cells has led to the identification more sophisticated and specified cancer therapies, it nevertheless remains imperative to execute the conventional therapies and the associated anticancer drug molecules. Various characteristic features like less toxicity and ability to induce an immune response make the therapy quite probable for various cancers like pancreatic, ovarian and Glioblastoma. Based on the results obtained from pre-clinical studies in model animals, it has set stage for the commercialization of the human trials. The advantages associated with this therapy for the treatment has started attracting focus to further modify and optimize the procedures for better results.
\end{abstract}

Keywords: Cancer; Therapeutic DNA, Gene therapy; Vector; Mutagenesis; Episomes; Glioblastoma

\section{Introduction}

Scientific arena has seen tremendous growth over the last few decades with special emphasis on the application of novel strategies to diagnose and treat medical ailments effectively. Of this diverse precinct, tissue engineering and nanotechnology plays an elemental role, as these two study areas have revolutionised current therapies to repair, replace cells, tissues and organs with the aid of various active molecules [1].

With recent advancements in biologics therapy and clinical therapy procedures, gene therapy remains as one of the most promising procedures that have been commercialized over the last few decades [2]. After its genesis, the first successful gene therapy was used in 1990 and since then number of clinical trials was conducted for it [3]. Only recently it was discovered that there is some sort of relationship between viruses and cancer and the root mechanism remained as one of the most important tools to study the cancer and its potential treatment [4].

\section{Gene therapy}

Gene therapy refers to the delivery of therapeutic DNA to treat an ailment. Gene therapy procedure requires the integration of a DNA into an artificially engineered virus that serves as a vector for the delivery of this DNA through the cells and ultimately the integration with the chromosome [5]. It is the treatment of genetic diseases by replacing the defective gene with a non-defective or functional gene. For the transfer of the foreign gene in this pathology, a vector or carrier is required that can transfer the gene into the host cells. The overall process of the viral integration with the cellular DNA for the delivery of the genetic material in the cell nucleus has been referred to as integration. This process may or may not alter the host cell's genetic material [6].

Cancer, being a genetic disease in nature, a proper understanding of genomics and proteomics is of utmost requirement in order to completely understand the genesis of the disease and its potential diagnosis [7].

\section{Classification of gene therapy}

Gene therapy can be broadly studied as germ-line and somatic gene therapies that can be applied to humans.

Germ line therapy requires the introduction of transgenic cells into the germ line and somatic cell line of the target population. The main punch of this type of therapy is that some of the genes can be carried forward to the gametes that may have the transgene. Germ line gene therapy has seen tremendous success with mice eggs. This therapy requires the removal of the blastocyst from the defective genotype and injecting the same with the transgenic cells with the wild type allele. Germ line gene therapy is still not yet commercialized for human trials nor does any official record of it exist.

Somatic gene therapy on the other hand does not include the genotype of the individual. It focuses only on the treatment of phenotype of the disease. Current trend of somatic therapy focuses on the introduction of the transgene into defective somatic cells either by using a disarmed retrovirus with the transgene being spliced into its genome or by the use of adenovirus. The action mechanism of this virus is well suited for epithelia cells and thereby injecting the genome in the cells. The major difference lies in the presence of the genome extra chromosomally. This property is specifically beneficial as it eliminates the possibility of insertional mutagenesis by the vector [8].

\section{Role of Gene Therapy in Cancer Studies}

Gene therapy has a vast range of applications not only restricted to

*Corresponding author: Irfan Ahmad, Amity Institute of Biotechnology, Amity University, Pin code- 201303, India, Tel: 8106564954; E-mail: bladesnyne@gmail.com

Received March 06, 2014; Accepted March 16, 2014; Published March 20, 2014

Citation: Ahmad I, Shahin R (2014) Autologous Gene Therapy - A Proactive Approach to Cancer Critique. J Cancer Sci Ther R1: 001. doi:10.4172/1948-5956. R1-001

Copyright: ( 2014 Ahmad I, et al. This is an open-access article distributed under the terms of the Creative Commons Attribution License, which permits unrestricted use, distribution, and reproduction in any medium, provided the original author and source are credited. 
the transient relief by suppressing the symptoms of the disease as most conventional drugs act. The procedure of curing disease by replacement of the defective gene with a functional has got a lot of prospects as far as the long term therapeutic advantage is concerned. The idea of gene therapy and its application has its roots in treatment of vision storage, haemophilia treatment and genetic causes of cancer related problems that too without the long repetitive drug application [9]. Moreover gene therapy offers a more viable and potentially less harmful method in local tumour control without the effects of the ionizing radiations. Radiotherapy albeit being a very probable method, effects like distant metastasis, recurrence does come into play due to the inhibition of DNA repair mechanisms, regulation of cell cycle and apoptosis [10].

\section{Edge over Conventional Therapies}

Although the conventional methods for treating the cancer cells are many, some if not all are coupled with a side effect that in the long run proves lethal to the patient [11]. Radiotherapy for cancer cells proved to be an effective way of eliminating cancer cells by targeting the tumour cells through radiation blast without focussing on the adjacent normal cells. However this therapy is not elemental as it also damages the normal undamaged cells. The implication of clinical gene therapy however works by "numerator to denominator of therapeutic ratio" concept where tumour cell kills outweigh the normal tissue damage [12].

\section{Gene Therapy Limitations}

Apart from the conventional cancer therapies, gene therapy also suffers from certain limitations that hinders with the overall method of its diagnosis. There are many genes that are being coded by both normal as well as cancer cells [13]. The malignant formation is not restricted to the activity of any single gene. On the contrary many genes are involved that results in the development of malignancy. The most important limitation that has put the application of this therapy at bay is being specific to a single gene product. As gene therapy targets are involved in multiple pathway of the metabolism, it becomes difficult to target all of these simultaneously [14]. Apart from this, cancer cells can avoid the gene therapy mechanisms just like viruses and bacteria. This property of these cancer cells to alter the mechanisms can be attributed to the "compensation" property of the cancer cells [9].

\section{Insertional mutagenesis}

The insertion or the delivery of the genetic material may induce insertional mutagenesis or oncogenic integration. Studies have revealed that there are certain vectors that behave differently while integrating with the start of the transcriptional units. Moloney Murine Leukaemia Virus (MLV) and others like Simian Immunodeficiency Virus (SIV) and Human Immunodeficiency Virus (HIV) did not exhibit the same behaviour. Such procedures may modify the gene expression at the insertion site or can also alter the gene product. Such kind of modifications may prove fatal as they can either give rise to the uncontrolled proliferation of the cells when the affected gene is the cancer gene [6].

\section{Toxicity}

Clinical trials of the gene therapy to cure cancer have seen tremendous applications in the last few decades. These trials although proved to be beneficial in some of the cases if not all, it does suffer from various safety risk issues. Notable among them is the high risk of integration at the random sites and toxicity. The most widely used vector for gene therapy trials, Adeno-Associated Virus (AAV) which has been widely introduced for Parkinson's disease, cystic fibrosis and haemophilia suffers from major drawbacks like random integration and low cloning capacity [15-18].

Episomal vectors of viral origin particularly of those from herpes simplex origin, although has a large cloning capacity and are equally capable of transducing wide variety of cells ranging from myoblasts, neurons and retinal cells, they also have the potential to integrating at random genetic sites in the genome [19-22].

\section{Phenotypic effects}

Systematic therapies available for the cancer diagnosis and prognosis have certain limitations during the whole course of the treatment. Effects like nausea, headache and infertility being the most common [23]. Cancer being genetic in nature has certain characteristics that are peculiar to the nature and type of the cancer. Asymptomatic progression being one, can be observed in brain neoplasms. A fast pace progression is noticed in pancreatic and ovarian cancers. These characteristics together with many more adds to the limitation of this systematic therapy which eventually results in high mortality rate $[24,25]$.

\section{Case Study}

Gene therapy offers wide range of therapies to detect cancer cells in vivo at very early stage of cancer development. One such case study is the detection of specific molecular abnormality.

Pancreatic cancer is one of the most arduous cancers to deal with. Due to its tendency to infiltrate and metastasize tissues from its early point of contact, it becomes difficult to track down the cancer from its development. Gene therapy plays a crucial role in detecting and disseminating the cancer in vivo by targeting specific molecular abnormality. Pancreatic cancer cells have a particular K-ras mutation addiction which is resistant in the normal cells. Non-cancerous cells appears to be resistant to suppression of K-ras mediated signalling by antisense K-ras RNA expression adenoviral vector [26].

\section{Targeting by vector}

The current intraperitoneal lipofection/polyfection methods, because of the low transduction efficiency, is not being put too much of the application. New strategies have been devised that requires more specific and active targeting mechanisms in the vector being used. Coxsackie Adenovirus Receptor (CAR) specificity of the fiber knows protein of the adenovirus vector proves elemental because of its high transduction efficiency and its specificity towards the target in vivo. Such vectors can be introduced via blood stream that redirects them to metastasis foci. Various ligands are being currently used that binds to the fiber knob of these vector and redirects them to the point of interest. Ligands such as integrin-binding proteins, phage-derived peptides, polylysine and proteoglycan-binding peptides are used to carry out the transport of these vectors to the desired location [27-29].

A rather more radical approach for targeting by vector mechanism makes use of the cell/tissue-specific promoters. Currently two promoters are being used, von Willebrand factor promoter to target endothelial cells [30] and modified rat probasin promoter for human prostate cancer cells [31]. These promoters work by either activating the Cre-recombinase enzyme that regulates the activity of the loxPregulated expression unit in the target cells or simply driving the sensitive cell-killing mechanism such as HSV-tk. 


\section{Non-viral episomes \& gene therapy}

Virus based vectors despite having advantages like high transduction and high efficiency factor, also suffers from major risk issues that might be lethal to the cell life. To name a few, insertional mutagenesis, cell transformation by viral proteins and innate immune response are the major probabilities. Gene therapy on a whole does provide a very reliable treatment for numerous cancer effects but these tendencies of virus based vectors cannot be ignored. To design a therapy with the same efficiency but lacking the aforementioned safety risks, non-viral vectors were designed. The major property of these non-viral vectors was the inability to integrate with the target chromosome and the absence of any viral element. These vectors were studied thoroughly and the version was modified with far better efficacy and increased safety guidelines that was visible in their stable transgene expression in the target cell [32].

\section{Glioblastoma - A special case of gene therapy}

Glioblastoma (GBM) because of its high heterogeneity and invasive nature remains as one of the most malignant type of the brain tumour. Although the standard therapies for GBM do sound effective but the effects of the therapy subsides after substantial amount of time [33].

The current therapy involves the use of alkylating drug Temozolomide (TMZ) [34] with intermittent conventional radiotherapy. The whole process is followed by the repeated administration of the adjuvant TMZ. The therapy fails to produce its long lasting effect and the relapse of the GBM is prone to occur. The inability of the therapy can be attributed to a number of factors including the DNA repair mechanism that imparts drug resistance to tumour cells [35-39].

Most of the gene therapy trials are directed to treat gliomas (GBM). This involves the delivery of nucleic acid which is either mediated by carrier molecules such as viruses, stem cells or lipid based nanoparticles or per se. The incorporation of unconjugated nucleic acids has been proved to be elemental in treatment of high grade gliomas [40]. One such case study was the involvement of phosphorothioate-modified antisense oligonucleotide that targets the mRNA for transforming factor-beta 2 (TGF- $\beta 2$ ). This protein molecule plays a very crucial role in the cell proliferation, invasion and metastasis of the cancer cells (gliomas) [33].

\section{Ovarian cancer \& transgenic expression}

Ovarian cancer originates in abdominal cavity and is one of the most difficult to diagnose at early levels of its course. It is usually diagnosed at advanced levels of clinical stages [40]. The main cause of the development of ovarian cancer is the over expression of Epidermal Growth Factor Receptor (EGFR) gene [41]. However in some cases, EGFR mutation deletion variant of type III is also recorded at III clinical stage. The EGFR gene upon sequencing revealed that its promoter region is devoid of TATA \& CAAT boxes [42].

The therapy used for advanced clinical level is systemic therapy but due to the various ill side effects from radiation, chemo, and radiation immunotherapy this therapy has very low therapeutic value [40]. Considering all the ill effects of the systemic therapies, it is the need of the hour to develop a therapy that would not only destroy the cancer cells but also should not be lethal to the normal healthy cells. In order to achieve that, bioengineered synthetic antibody guided vectors were prepared that are capable of carrying recombinant DNA constructs for human rDNase and multiple transgene [43].

\section{Bioengineered vectors and transgenic expression}

Non-cancerous tissue from patients suffering from cancer of ovary, liver etc. was isolated and was subjected to nucleic acid extractor for genomic DNA isolation. mRNA was subsequently isolated using trizol reagent. The cDNA was regenerated using random hexamers and the enzyme reverse transcriptase. Coding sequences were essentially imported from NCBI and are used to design primers using PrimerBlast for DNASE1, DNASE1L3, DNASE2 and DFFB. The coding sequence was also imported for EGFR and was synthesized on the DNA synthesizer.

These DNases were integrated to the transfection vectors. After the binding of these DNases to the DNA constructs, they were docked to the synthetic antibodies carrying the biotin tag. These biotags aids in guiding these vectors carrying transgenes to the target cell. The transgene once transfected into the target cell, its expression was determined using MPFS and electrophoresis of the nucleus lysate. The entire course of this therapy using biotags was studies using techniques such as NMRS or FCM that quantifies the apoptotic cells and to also confirm about the confirmation of these cells as viable or dead $[40,44]$.

This whole strategy can be summed up as:

- Bioengineered vectors were created for carrying suicide genes which are guided by antibodies for EGFR.

- Genetically engineered DNA constructs for human rDNASE1, DNASE1L3, DNASE2 and DFFP. These all are controlled by EGFR promoter region.

- Intracellular targeting of the expressed transgenic DNases to destroy the ovarian cancer cells [40].

\section{Delivery Molecules for Gene Therapy}

The multiple advantages of non-viral vectors has attracted special focus to derive more sophisticated molecules that can be used for the appropriate delivery of the nucleic acids without imposing the threats of the conventional viral vectors. These non-viral vectors owe its therapeutic advantage to the properties possessed such as low toxicity and comparatively low immunogenicity. Apart from this the presence of the physicochemical versatility that enables these molecules to modify their surface for targeting specific cells/tissues [45,46].

\section{Lipid-based nanoparticles}

Design of these carrier molecules ranges from dendrimers, nanogold, liposomes, nanoemulsions, drug-carrier conjugates etc. are based upon the assemblies of naturally/synthetically occurring components [47]. Lipid based nanoparticles are very promising in the delivery of nucleic acids at the desired region. One of the best activity can be observed with liposome-mediated intratumoral delivery of genes that code for interferon proteins, IFN- $\beta$ which in turn generates an anti tumoral activity $[48,49]$. Similarly cationic liposomes carrying the IFB- $\beta$ gene into the tumour region of the patients has resulted in a considerable decrease in the tumour volume [50]. Moreover the incorporation of the gene was well endured. This study was conducted on a very small scale thus no significant conclusion was drawn from it. However such therapy can be studied to determine the immunogene response on a large scale that can be effectively used for GBM.

\section{Polymer based carrier systems}

Polymer based nanoparticles finds application in anticancer 
therapies because of their multifunctionality and specificity. These molecules can carry nucleoside analogues that are very effective in anticancer procedures. Nucleoside analogues because of the antimetabolite nature and their ability to interfere with the synthesis of nucleic acids in cellular and cancer cell systems, there is an immediate need to design molecules that can effectively carry these analogues to the desired location. Usually a high dose of these nucleoside analogues is required because of their nonspecific distribution as well as rapid clearance from the system.

Depending upon the type of administration, various polymer based nanoparticles are designed that are non-stringent to cancer therapies but are also used for anti-HIV agents. Poly (alkyl-cyanoacrylate) nanoparticles are particularly used for anticancer therapies and are usually administered either orally or intravenously. Albumin on the other hand has been found to be extremely promising for designing of the carrier molecules but is restricted only for oral administration [51].

These polymer based nanoparticles are extensively used in animal models for anti-cancer therapies [33]. Polyethylene glycolpolyethylenimine (PEG-PEI) carrier molecule targets the cancer cell by coupling with cyclic arginine-glycine aspartic acid-D-tyrosine-lysine peptide (cRGD). This peptide binds to a protein molecule integrin, $\alpha \mathrm{V} \beta 3$ that is overexpressed in the cells and is actively engaged in invasion and angiogenesis [52].

These nanoparticles tend to increase the intracellular accumulation of the therapeutic agent at the cancer juncture either by passive way or targeting or active. Either way there seems to be low level toxicity in the normal cells. These targeted nanoparticles can also be modified by enabling them to be $\mathrm{pH}$-sensitive or temperature sensitive carriers. Such a procedure has its own advantages as it ensures the delivery of the therapeutic agent at the proper site within the microenvironment [53].

\section{Neural \& mesenchymal stem cells as carrier molecules}

Stem cells have tremendous application as carrier molecules because of their highly specific nature to target cancer cells (gliomas) [54]. The underlying concept is the secretion of various cytokines and growth factors such as Vascular Endothelial (VEGF) and PlateletDerived Growth Factors (PDGF). These molecules acts as chemo attractants that drives the migration of these cells towards the target tumour [55,56]. Neural and mesenchymal stem cells in particular, are being generally employed for the aforementioned procedure. Adipose tissue and bone marrow are very rich source of Mesenchymal Stem Cells (MCS's) whereas both adult and foetal brain are good source of neural stem cells $[57,58]$.

Mesenchymal stem cells have certain advantages that enable them to be used as a most efficient therapeutic delivery agent. The tumortropic property of these cells makes them accumulated at the cancer site thereby enabling better delivery of the therapeutic agent. The unique ability to modulate the immune response increases the possibility of survival of MCS's. Moreover the potential application of using autologous stem cells minimizes the chances of immune response, if any, thereby increasing the cell survival [59].

\section{Gene Therapy Perspectives}

Gene therapy holds a great deal of promise in various autosomal, $\mathrm{X}$-linked genetic disorders as well. Retinitis Pigmentosa is a form of heterogeneous genetic disorder that can be transferred via $\mathrm{x}$-linked or mitochondria. X-linked retinitis pigmentosa is the most severe form of disorder and is caused by the mutation in the gene that regulates the activity, Retinitis Pigmentosa GTPase Regulator (RPGR). This gene is mostly expressed in photoreceptors in the basal body of the cilia. The most commonly used therapy accounts for the application of retinal gene delivery. Vectors that are commonly used for transfer procedures are Adeno-Associated Virus (AAV) [60].

Gene therapy prognosis can be efficiently assayed and studied by the use of Magnetic Resonance Imaging (MRI) techniques. Various magnetic nanoparticles that are modified with polymers can be effectively designed to carry fluorescent molecules to target specific cancer cells such as Antigreen Fluorescent Protein (anti-GFP) siRNAs in rat gliomas cells. Such carrier molecules were reported to enhance the MRI thereby further increasing the possibility of monitoring glioma treatment [33].

\section{Conclusions}

Gene therapy has been discovered as an elemental aspect in the treatment and studying the course of the cancer, the various causes and the molecules that are known to be involved directly or indirectly in its causation. Many clinical trials have been performed over the last few years that have turned out to be quite promising in achieving the desired goal. These therapies not only have eliminated the detrimental effects of the conventional procedures but also highlighted the future prospects to treat the disease enormously. Subsequently more and more research is being carried out that emphasizes the role of the transfection vectors and how they are involved in guiding the target gene to its destination. Further insights into the biochemistry and molecular studies on the molecules associated with cancer and their regulation will further advance out understanding of the therapy and also to create novel therapy procedures either by optimizing the current scheme or by developing an entirely new cancer therapy. The study faces numerous technical challenges, not the least of which is the establishment of an appropriate gene carrier that does not interfere with the normal healthy cells. Efforts to address these issues are underway, and if past success is any indication, this technology is certainly one that will have a major impact in future health care practice.

\section{References}

1. Kingsley JD, Ranjan S, Dasgupta N, Saha P (2013) Nanotechnology for tissue engineering: Need, techniques and applications. J Pharm Res 7: 200-204

2. Ledley FD, McNamee LM, Uzdil V, Morgan IW (2013) Why commercialization of gene therapy stalled; examining the life cycles of gene therapy technologies. Gene Therapy 21: 188-194

3. Gene Therapy Clinical Trials Wordlide (2014) Journal of Gene Medicine. Wiley \& Sons, New Jersey, USA.

4. Francisco AG (2012) There is Crosstalk between Human Papillomavirus and Cigarette Smoke Components for Cancer Development? J Carcinogene Mutagene 3: e105

5. Genetics Home Reference (2014) How does gene therapy work? Lister Hill National Center for Biomedical Communications, USA.

6. Alessandro A, Di Serio C (2009) Vectors and Integration in Gene Therapy: Statistical Considerations. J Comput Sci Syst Biol 2: 117-123.

7. Malecki M (2012) Cancer Suicide Gene Therapy. J Genet Syndr Gene Ther 3: e114

8. Griffiths AJF, Miller JH, Suzuki DT (2000) Gene Therapy - An Introduction to Genetic Analysis (7th edn.). W. H. Freeman, New York, USA

9. Bosiack AP, Giuliano EA, Mohan RR (2012) Corneal Gene Therapy in Veterinary Medicine: A Review. J Veterinar Sci Technol S8:001. 
10. Kaliberov SA, Buchsbaum DJ (2012) Chapter Seven - Cancer Treatment with Gene Therapy and Radiation Therapy. Adv Cancer Res 115: 221-263.

11. Khan DR (2010) The use of Nanocarriers for Drug Delivery in Cancer Therapy J Cancer Sci Ther 2: 058-062.

12. Greenberger JS, Epperly MW (2014) Chapter 9-Gene Therapy in Radiotherapy of Cancer. Gene Ther Cancer 123-133.

13. Rubenstein M, Hollowell CMP, Guinan P (2012) Tumor Compensation Associated with Gene Therapy. J Genet Syndr Gene Ther 3:e117.

14. Rubenstein M, Dunea G, Guinan P (1994) Growth factor deprivation therapy utilizing antisense oligonucleotides. Drug News and Perspectives 7: 517-524.

15. During MJ, Kaplitt MG, Stern MB, Eidelberg D (2001) Subthalamic GAD gene transfer in Parkinson disease patients who are candidates for deep brain stimulation. Hum Gene Ther 12: 1589-1591.

16. Flotte TR, Zeitlin PL, Reynolds TC, Heald AE, Pedersen P, et al. (2003) Phase I trial of intranasal and endobronchial administration of a recombinantadenoassociated virus serotype 2 (rAAV2)-CFTR vector in adult cystic fibrosis patients: a two-part clinical study. Hum Gene Ther 14: 1079-1088.

17. Manno CS, Chew AJ, Hutchison S, Larson PJ, Herzog RW, et al. (2003) AAV mediated factor IX gene transfer to skeletal muscle in patients with severe hemophilia B. Blood 101: 2963-2972.

18. Nakai H, Yant SR, Storm TA, Fuess S, Meuse L, et al. (2001) Extrachromosomal recombinant adeno-associated virus vector genomes are primarily responsible for stable liver transduction in vivo. J Virol 75: 6969-6976.

19. Wang Y, Mukherjee S, Fraefel C, Breakefield XO, Allen PD (2002) Herpes simplex virus type 1 amplicon vector-mediated gene transfer to muscle. Hum Gene Ther 13: 261-273.

20. Fraefel C, Mendes-Madeira A, Mabon O, Lefebvre A, Le Meur G, et al. (2005) In vivo gene transfer to the rat retina using herpes simplex virus type 1 (HSV1)-based amplicon vectors. Gene Ther 12: 1283-1288.

21. Muller L, Saydam O, Saeki Y, Heid I, Fraefel C (2005) Gene transfer into hepatocytes mediated by herpes simplex virus-Epstein-Barr virus hybrid amplicons. J Virol Methods 123: 65-72.

22. Berges BK, Wolfe JH, Fraser NW (2007) Transduction of brain by herpes simplex virus vectors. Mol Ther 15: 20-29.

23. Malecki M (2012) Frontiers in Suicide Gene Therapy of Cancer. J Genet Syndr Gene Ther 3:119.

24. American Cancer Society (2012) Cancer Facts and Figures, USA

25. Jemal A, Siegel R, Xu J, Ward E (2010) Cancer statistics, 2010. CA Cancer J Clin 60: 277-300.

26. Yoshida T, Obnami S, Aoki K (2004) Development of gene therapy to target pancreatic cancer. Cancer Sci 95: 283-289.

27. Krasnykh V, Douglas JT (2002) Targeted adenoviral vectors I: transductional targeting. In: Curiel DT, Douglas JT. Adenoviral vectors for gene therapy. San Diego: Academic Press, USA.

28. Nicklin SA, White SJ, Watkins SJ, Hawkins RE, Baker AH (2000) Selective targeting of gene transfer to vascular endothelial cells by use of peptides isolated by phage display. Circulation 102: 231-7.

29. Belousova N, Krendelchtchikova V, Curiel DT, Krasnykh V (2002) Modulation of adenovirus vector tropism via incorporation of polypeptide ligands into the fiber protein. J Virol 76: 8621-8631.

30. Ozaki K, Yoshida T, Ide H, Saito I, Ikeda Y, et al. (1996) Use of von Willebrand factor promoter to transducer suicidal gene to human endothelial cells, HuVEC Hum Gene Ther 7: 1483-1490.

31. Furuhata S, Ide H, Miura Y, Yoshida T, Aoki K (2003) Development of a prostate- specific promoter for gene therapy against androgen-independent prostate cancer. Mol Ther 3: 366-374

32. Claudia H, Hans JL (2011) pEPI for Gene Therapy: Non-viral episomes and their Application in Somatic Gene Therapy. J Cell Sci Ther S6-001.

33. Pedro MC, Maria CP (2013) Viral and Non-Viral Gene Therapy for Glioblastoma: New Insights into the Treatment of Malignant Brain Tumors. J Genet Syndr Gene Ther 4: 7.

34. Stupp R, Mason WP, van den Bent MJ, Weller M, Fisher B, et al. (2005)
Radiotherapy plus concomitant and adjuvant temozolomide for glioblastoma. N Engl J Med 352: 987-996.

35. Dunn GP, Rinne ML, Wykosky J, Genovese G, Quayle SN, et al. (2012) Emerging insights into the molecular and cellular basis of glioblastoma. Genes Dev 26: 756-784.

36. Stupp R, Hegi ME, Mason WP, van den Bent MJ, Taphoorn MJ, et al. (2009) Effects of radiotherapy with concomitant and adjuvant temozolomide versus radiotherapy alone on survival in glioblastoma in a randomised phase III study: 5-year analysis of the EORTC-NCIC trial. Lancet Oncol 10: 459-466.

37. Agnihotri S, Gajadhar AS, Ternamian C, Gorlia T, Diefes KL, et al. (2012) Alkylpurine-DNA-N glycosylase confers resistance to temozolomide in xenograft models of glioblastoma multiforme and is associated with poor survival in patients. J Clin Invest 122: 253-266.

38. Cahill DP, Levine KK, Betensky RA, Codd PJ, Romany CA, et al. (2007) Loss of the mismatch repair protein MSH6 in human glioblastomas is associated with tumor progression during temozolomide treatment. Clin Cancer Res 13 2038-2045.

39. Kaina B, Christmann M, Naumann S, Roos WP (2007) MGMT: key node in the battle against genotoxicity, carcinogenicity and apoptosis induced by alkylating agents. DNA Repair (Amst) 6: 1079-1099.

40. Malecki M, Dahlke J, Haig M, Wohlwend L, Malecki R (2013) Eradication of Human Ovarian Cancer Cells by Transgenic Expression of Recombinant DNASE1, DNASE1L3, DNASE2, and DFFB Controlled by EGFR Promoter: Novel Strategy for Targeted Therapy of Cancer. J Genet Syndr Gene Ther 4: 152.

41. Moscatello DK, Holgado-Madruga M, Godwin AK, Ramirez G, Gunn G (1995) Frequent expression of a mutant epidermal growth factor receptor in multiple human tumors. Cancer Res 55: 5536-5539.

42. Ishii S, Xu YH, Stratton RH, Roe BA, Merlino GT, et al. (1985) Characterization and sequence of the promoter region of the human epidermal growth factor receptor gene. Proc Natl Acad Sci U S A 82: 4920-4924.

43. Malecki M, Szybalski W (2012) Isolation of single, intact chromosomes from single, selected ovarian cancer cells for in situ hybridization and next generation sequencing. Gene 493: 132-139.

44. Malecki M, Malecki B (2012) Routing of biomolecules and transgenes' vectors in nuclei of oocytes. J Fertili In Vitro 108: 108-118.

45. Yoshida J, Mizuno M (2003) Clinical gene therapy for brain tumors. Liposoma delivery of anticancer molecule to glioma. J Neurooncol 65: 261-267.

46. Zhou J, Atsina KB, Himes BT, Strohbehn GW, Saltzman WM (2012) Novel delivery strategies for glioblastoma. Cancer J 18: 89-99.

47. Puri A, Loomis K, Smith B, Lee JH, Yavlovich A, et al. (2009) Lipid-based nanoparticles as pharmaceutical drug carriers: from concepts to clinic. Crit Rev Ther Drug Carrier Syst 26: 523-580.

48. Natsume A, Mizuno M, Ryuke Y, Yoshida J (1999) Antitumor effect and cellular immunity activation by murine interferon-beta gene transfer against intracerebral glioma in mouse. Gene Ther 6: 1626-1633.

49. Natsume A, Tsujimura K, Mizuno M, Takahashi T, Yoshida J (2000) IFN-beta gene therapy induces systemic antitumor immunity against malignant glioma. J Neurooncol 47: 117-124.

50. Yoshida J, Mizuno M, Fujii M, Kajita Y, Nakahara N, et al. (2004) Human gene therapy for malignant gliomas (glioblastoma multiforme and anaplastic astrocytoma) by in vivo transduction with human interferon beta gene using cationic liposomes. Hum Gene Ther 15: 77-86.

51. Hillaireau H, Le Doan T, Couvreur P (2006) Polymer-based nanoparticles fo the delivery of nucleoside analogues. J Nanosci Nanotechnol 6: 2608-2617.

52. Zhan C, Wei X, Qian J, Feng L, Zhu J, et al. (2012) Co-delivery of TRAIL gene enhances the anti-glioblastoma effect of paclitaxel in vitro and in vivo. $\mathrm{J}$ Control Release 160: 630-636

53. Nguyen KT (2011) Targeted Nanoparticles for Cancer Therapy: Promises and Challenges. J Nanomedic Nanotechnol 2: 103e.

54. Hoshino G, Yagi H, Hasegawa H, Ishii Y, Okabayashi K, et al. (2013) Human Mesenchymal Stem Cells Migrate toward Colon Cancer Partially Regulated by HMGB1. J Cell Sci Ther 4: 145.

55. Cheng P, Gao ZQ, Liu YH, Xue YX (2009) Platelet-derived growth factor BB 
Citation: Ahmad I, Shahin R (2014) Autologous Gene Therapy - A Proactive Approach to Cancer Critique. J Cancer Sci Ther R1: 001. doi:10.4172/19485956.R1-001

promotes the migration of bone marrow-derived mesenchymal stem cells towards C6 glioma and up-regulates the expression of intracellular adhesion molecule-1. Neurosci Lett 451: 52-56.

56. Schmidt NO, Przylecki W, Yang W, Ziu M, Teng Y, et al. (2005) Brain tumo tropism of transplanted human neural stem cells is induced by vascular endothelial growth factor. Neoplasia 7: 623-629.

57. Tocci A, Forte L (2003) Mesenchymal stem cell: use and perspectives. Hematol J: $92-96$.
58. Zuk PA, Zhu M, Ashjian P, De Ugarte DA, Huang Jl, et al. (2002) Human adipose tissue is a source of multipotent stem cells. Mol Biol Cell 13: 42794295.

59. Nguyen KT (2013) Mesenchymal Stem Cells as Targeted Cell Vehicles to Deliver Drug-loaded Nanoparticles for Cancer Therapy. J Nanomed Nanotecho 4: e128.

60. Shu X (2013) Gene Therapy for X-Linked Retinitis Pigmentosa. Clon Transgen 3: e108. 